\title{
Review of Multibody Simulation Modelling of Gear Systems subjected to Uncertainties
}

\section{Modélisation et Simulation Multi-corps des Systèmes d'engrenages avec prise en compte des incertitudes}

\author{
C V S R Subrahmanyam ${ }^{1}$ \\ ${ }^{1}$ Associate Professor, Dept of Mechanical Engineering, Tolani Maritime Institute, Pune, cvsrs1968@gmail.com
}

ABSTRACT. Review of the capabilities of multibody simulation models and their short comings are discussed in this article. Finally an attempt was made to evolve a methodology which leads to dynamically efficient robust design of mechanical systems like gear box.

KEYWORDS. Multibody Systems, Simulation, Robust Design, Gear box.

\section{Introduction}

Generally speaking, multibody systems can be classified as rigid multibody systems or flexible multibody systems. Rigid multibody systems are assumed to consist only of rigid bodies. These bodies, however, may be connected by massless springs, dampers, and/or actuators. This means that when rigid multibody systems are considered, the only components that have inertia are assumed to be rigid bodies. Flexible multibody systems, on the other hand, contain rigid

and deformable bodies. Deformable bodies have distributed inertia and elasticity which depend on the body deformations. As the deformable body moves, its shape changes and its inertia and elastic properties become functions of time. For this reason, the analysis of deformable bodies is more difficult than rigid body analysis[1].

There is plenty of software tools specialized in the field of multibody dynamics, which can be currently used for the solution of the engineering or research problems (ADAMS, SIMPACK, alaska, ...). The equations of motion while working with these tools are created on the basis

of so called multibody formalisms. They are special proposed algorithms for the automatic generation of equations of motion of the coupled rigid body systems. Also flexible bodies can be incorporated in computational models. The software tools integrate more or less comfortable pre- and post-processing environment with efficient solvers developed by groups of mathematicians and engineers. On the other hand, it is sometimes more advantageous to formulate and to solve the equations of motion of a studied multibody system without usage of the commercial software tools. The main reason is the black-box-like behavior of the commercial tools and the limited possibility of introducing some special features and special model elements as well as some non-standard solution or optimization methods[2].

Computational methods are used extensively for the analysis and design of engineering dynamic systems such as automobiles, aircrafts and wind-turbines. Credible computational methods can lessen 
the need for expensive experimental analysis and prototype building and reduce the cost of development of these products. Some engineers believe that the credibility of computational predictions can be enhanced only by using intensive numerical approaches, e.g., by increasing the mesh density in the finite element model of a structure. While this may be necessary for some applications (e.g., modelling the tip of a crack) it is not sufficient to improve the credibility of computational prediction. This is due to the fact that several types of uncertainties exist in the whole process of science-based computational predictions as shown in the figure below. Such uncertainties include (a) parametric uncertainty (e.g., uncertainty in geometric parameters, friction coefficient, viscosity, strength of the materials involved), and (b) nonparametric uncertainty such as those arising from model inadequacy (e.g., using linear law when it is actually nonlinear) and low fidelity models (e.g., damping, multi-scale issues, damage mechanisms). These uncertainties must be assessed and managed for reliable computational prediction

\section{Sources of parametric uncertainties}

(i)- Uncertainties for the spatial mass distribution inside a body. For instance, such a type of uncertainties can be encountered for a vehicle in which the passengers have a mass and a position that are variable. For each body, this type of uncertainties yields a random mass, a random position of the center of mass, and a random tensor of inertia.

Consequently, the mass matrix $[\mathrm{M}]$ and the Coriolis forces $\mathrm{K}$ are random. The probability density functions (pdf) of these random masses, random positions of the centers of mass, and random tensors of inertia have been constructed 1 using the Maximum Entropy Principle, in which a special care has been devoted to the probabilistic modeling of the random tensor of inertia in following the methodology of the nonparametric probabilistic approach of uncertainties introduced in 23,24 for the construction of random matrices.

(ii)- Uncertainties in the joints. For the ideal joints, the directions and the points defining the joints can be uncertain.

These uncertainties may be due to manufacturing tolerances, or due to the natural wear during the life cycle of the multibody system. Such uncertainties have to be taken into account in order to ensure a good accuracy for the prediction of the dynamical response of the multibody system. For non-ideal joints the friction coefficients can also be uncertain. The randomness in the joints between the bodies yields a random constraint vector.

(iii)- Uncertainties in internal forces. Concerning internal forces, there may be uncertainties in the constitutive laws of the multidimensional springs and dampers. In such a case, uncertainties may be taken into account in the parameters of the constitutive laws, or directly in the stiffness and damping matrices using the nonparametric probabilistic approach of uncertainties. [3]

\section{Model Uncertainty Questions}

What is the most appropriate approach to consistently represent and reason about uncertainty in complex systems consistently?

What is the best approach to characterizing the uncertainty associated with a simulation model in order to enable and facilitate reuse? 
How should one aggregate knowledge, expertise, and beliefs of multiple experts across different domains?

What is the best approach to take advantage of the large and diverse datasets for characterizing uncertainty and for improving model accuracy?

What are the most promising approaches to accelerate the validation of models for specific application contexts?

\section{Research Challenges:}

A common problem in the numerical simulation of real-world systems is the fact that exact values for the parameters of the models can exhibit a high level of uncertainty. This non-determinism in numerical models may arise as a consequence of different sources, motivating some categorization of uncertainties.

Although other classifications are possible in almost the same manner, the following categorization proves to be well-suited in this context: aleatory uncertainties, such as natural variability or scatter, on the one side, and on the other side, epistemic uncertainties, which arise from an absence of information, rare data, vagueness in parameter definition, subjectivity in numerical implementation, or simplification and idealization processes employed in the modeling procedure.

All these conditions manifest as uncertain model parameters and in some situations as uncertain initial or boundary conditions. Consequently, the results that are obtained for simulations that only use one specific set of values as the most likely ones for the model parameters cannot be considered as representative of the whole spectrum of possible model configurations.

Furthermore, this fake exactness provided by the numerical simulation of models with uncertain but exact-valued parameters can significantly affect the comparison between numerical simulations and experimental testing.

Namely, such a comparison may be rated as unsatisfactory if the crisp-valued simulation results do not well match the experimental ones, even though it might be absolutely satisfactory, if the uncertainties inherent to the models would have been appropriately taken into account in the simulation procedure

\section{Approaches for Analysis:}

Stochastic analysis: the stochastic approach to uncertain problems is to model the structural parameters as random quantities. Therefore, all information about the structural parameters is provided by the probability density functions. This probability density function is then used to determine an estimate of the system's behavior.

Fuzzy analyses: The fuzzy approach to the uncertain problems is to model the structural parameters as fuzzy quantities. In conventional set theories, either an element belongs or does not belong to set. However, fuzzy sets have a membership function that allows for "partial membership" in the set. Using this method, structural parameters are quantified by fuzzy sets. 
Interval analysis: The interval approach to the uncertain problems is to model the structural parameters as interval quantities. In this method, the uncertainty in the elements is viewed by a closed set-representation of element parameter that can vary within intervals between extreme values. Then, structural analysis is performed using interval operations.[3]

\section{Case Study: Proposed Methodology to fix uncertainty in Multibody Simulation Model of Gearbox.}

Industrial gear systems produce noise when they are subjected to different operating conditions. That is to say that the final design of gear box is validated for a set of given operating conditions. When the gear box is subjected to any other operating condition it gives rise to noise signatures.

Now a day many attempts were made to make the design robust. In order to make the final design of the gear box robust, consider the final design of gear box. Collect the noise signature while testing.

Generate a multibody simulation model with the help of software like Pro Engineer/Ansys. Estimation of effect of parameters like mass, stiffness and damping on dynamic performance of the simulation is to be conducted. Sensitivity analysis was then conducted to identify critical parameters, with ranking of their contributions to the variability in the performance parameter. This information is useful to improve the efficiency of probabilistic analysis and to guide efforts in data collection. Further analysis to identify an approximation function, called a Response Surface Function (RSF) was carried out.

Compare the noise signature of the gear box with expected dynamic performance characteristics.

The difference in the two signatures is to be considered as Model Based Epistemic Uncertainty.

Now Consider the parameters of the simulation model: mass, stiffness and damping.

Set the variation ranges for these parameters.

Research techniques like Design of Experiments can be applied for conducting and concluding the possible ranges for the above parameters to fix the noise signatures and arrive at robust dynamic performance characteristics. Software MSC.ADAMS has probabilistic design

capabilities such as Design of Experiments (DOE) and Monte Carlo simulation (MCS). These

models were used to predict how the variability in the input parameters affects the variability

Then between the concluded ranges of the parameters optimization study can be conducted to make the simulation model dynamically efficient.

By adopting the above methodology and using MATLAB software, it is possible to achieve dynamically efficient robust design of the gear box.

\section{Conclusion}

Role of Uncertainties in Multibody Simulation Modelling has been explored in this article. A methodology is proposed to characterize the uncertainties in the process of evolving a robust design of a gearbox. 


\section{Declarations}

This article is not a part of any project, hence no funding is received. Proposed Methodology is the author's contribution in the paper. Author would like to acknowledge Dr. Nitin Khedkar, Professor at Symbiosis Institute of Technology for his valuable time during the discussions.

\section{References}

[1] Computational Dynamics, Second Edition, Ahmed A. Shabana

[2] Application of Stabilization techniques in the dynamic analysis of multibody systems, M Hajzman, P Polach,2007, Applied and Computational Mechanics,479-488

[3] Dynamic Response bound estimation of Structures with Interval Parameters, Janaina CV Albuquerque, Jose Juliano De L Junior,2018, Journal of Applied Mechanical Engineering,

[4] Design and Dynamics Modelling for Electric Vehicle. Maria Tomosikova, Frantisek Brumercik,Aleksander Nieoczym, 2017,Applied Computer Science, Vol.13, No. 3, pp 19-31

[5] Simulation and Experimental Research on Vibration and Noise of Transmission, Yulong Lei, Bo Yan, Yao Fu, Liu Zongsheng, Chen Wei \& Hou Liguo, 2012, Advances in Engineering Research, 4th International Conference on Renewable Energy and Environmental Technology (ICREET 2016)

[6] NVH Potential of PM Gears for Electrified Drivetrains, Dr. Gerd Kotthoff, 2018. Analysis of Excitation Behavior of Two-Stage Gearbox Based Upon Validated Simulation Model, Christian Brecher, Christoph Löpenhaus and Marius Schroers, 2017

[7] Gearbox Transmission Error study using Multi Body Analysis software MSC Adams, Antonio Roldán Sánchez, 2018, Dpto. Ingeniería y Ciencia de los Materiales y del Transporte Escuela Técnica Superior de Ingeniería Universidad de Sevilla Sevilla, 2018

[8] Multibody dynamic analysis of a gear transmission system in electric vehicle using hybrid user-defined elements, Chao Liu, Zong-de Fang,Xuan Liu and Sheng-yang Hu, 2018, Proc IMechE Part K:J Multi-body Dynamics,0(0) 1-13

[9] Design and Study of Transmission System for Electric Vehicles, Ashwin Chander, Madan Kumar, Shobith Nambiar, Harsh Zaveri,2018, IJESC, Vol.8, No. 3 